\title{
Double Truncated Transmuted Fréchet Distribution: Properties
}

\section{and Applications}

\author{
Muhammad Zafar Iqbal ${ }^{1}$, Muhammad Zeshan Arshad ${ }^{2}$, Munir Ahmad ${ }^{3}$, Iftikhar Ahmad ${ }^{4}$, Taswar Iqbal ${ }^{5}$ \\ Muhammad Arslan Bhatti ${ }^{6}$ \\ 1, 5, 6 Department of Mathematics and Statistics, University of Agriculture, Faisalabad, Pakistan \\ ${ }^{2,3}$ National College of Business Administration and Economics, Lahore, Pakistan \\ ${ }^{4}$ University of Gothenburg, Department Mathematical Sciences, Sweden \\ E-mails:*mzts2004@hotmail.com, profarshad@yahoo.com, munirahmaddr@yahoo.co.uk, \\ ifti_ch2002@yahoo.com, therisers1@gmail.com and bhatti.stat.agri@gmail.com
}

\begin{abstract}
In this paper, we modify the Mahmoud and Mandouh (2013) model by adopting double truncation technique. It is referred to as Double Truncated Transmuted Fréchet (DTTF) distribution. Diverse probabilistic and reliability measures are developed and discussed. The MLEs of parameters are derived and a simulation study is also made. The DTTF distribution is modeled by two real-time datasets and supportive rationalized results provide the evidence that DTTF distribution is a reasonably better fit model than its competing models.
\end{abstract}

Keywords: Fréchet Distribution, Double Truncation, Hazard Function, Moments, MLE, Quadratic Rank Transmutation Map (QRTM), Rényi entropy, Order Statistics.

DOI: $10.7176 / \mathrm{MTM} / 9-3-02$

Publication date: March $31^{\text {st }} 2019$

\section{Introduction}

In numerous continuous probability distributions, particularly extreme value theory is an important part of statistical literature and one of the special cases (inverse Weibull, inverse Rayleigh, inverse Exponential or Gumbel type-II) is Fréchet distribution. Maurice Frechet (1878-1973) a French mathematician developed a significant relation with Pareto distribution in 1927 when he discovered a limiting distribution for higher order statistic. The vital role of Fréchet distribution is observed in applied fields, for instance, through accelerated lifetesting to engineering, geology, hydrology, horse racing, insurance, meteorology, sea currents, wind speed and many other diverse problems of life. Several generalizations and modifications, as well as progressive expansions over the last two decades, have been studied and a lot more is about to happen.

Nadarajah and Kotz (2003) developed an Exponentiated Fréchet distribution. Krishna et al. (2013) developed Fréchet distribution in Marshall-Olkin family of distributions and discovered its application in time series modeling. Mead and Abd-Eltawab (2014) expressed the Fréchet distribution in Kumaraswamy family of distributions and illustrated its application in the breaking stress of carbon fibers and strength of $1.5 \mathrm{~cm}$ glass fibers datasets. Afify et al. (2016a) developed Marshall-Olkin Fréchet distribution in Kumaraswamy family of distributions and discussed its application in survival times of guinea pigs and the strength of $1.5 \mathrm{~cm}$ glass fibers datasets. Transmuted Marshall Oklin Fréchet distribution introduced in Kumaraswamy family of distributions by Yousof et al. (2016). They developed its application in carbon fiber and glass fiber datasets. Mead et al. (2017) expressed the Exponentiated Fréchet distribution in the beta distribution and discovered its application in the Myelogeneous Leukemia and carbon fiber datasets. Mansour et al. (2018) illustrated the Exponentiated Fréchet distribution in Kumaraswamy family of distributions. They studied its application in the strength of $1.5 \mathrm{~cm}$ glass 
fibers dataset. Mansour et al. (2018) this time exponentiated the Marshall-Olkin Fréchet distribution and discovered its application in Myelogeneous Leukemia and flood peaks datasets. Mansour et al. (2018) generalized the Fréchet distribution in Odd Lindley family of distributions. They illustrated its application in exceedances of flood peaks and the breaking stress of carbon fibers datasets. Oguntunde et al. (2019) developed a compound of the Gompertz and Fréchet distribution. They developed its application in strength of carbon fibers and hailing times datasets.

Quadratic Rank Transmutation Map (QRTM) as a generator first time introduced by Shaw and Buckley (2009) for non-Gaussian distributions by adding a new parameter $\lambda$ to base distribution $G(x)$. CDF of QRTM is followed by

$$
F(x)=(1+\lambda) G(x)-\lambda G^{2}(x) \quad, \text { for }|\lambda|<1
$$

For $\lambda=0, F(x)=G(x)$, where $G(x)$ is base distribution.

Scientific literature extended by Mahmoud and Mandouh (2013) when they generalized the Fréchet distribution in QRTM family of distributions and developed its application in breaking stress of carbon fiber and simulated datasets. The CDF of TFD is

$$
F(x)=(1+\lambda) e^{-\alpha x^{-\beta}}-\lambda e^{-2 \alpha x^{-\beta}}, \text { for } \alpha, \beta>0 \text { and }|\lambda|<1 \text {. }
$$

Afify et al. (2015) investigated Marshall Olkin Fréchet distribution in QRTM family of distributions and discussed its application in breaking stress of carbon fibers and strength of $1.5 \mathrm{~cm}$ glass fibers datasets. Arshad et al. (2018) generalized Exponentiated Moment Pareto in QRTM and found its application in four-lifetime datasets. Abayomia and Adeleke (2019) developed the transmuted edition of half normal distribution and discussed its application in purchasing behavior of customers from a wholesale outlet. Bhatti and Ali (2019) developed a range of characterizations of the transmuted edition of Exponentiated Pareto-I distribution.

In distribution theory, truncation is referred to as conditional distribution that provides more constructive and reliable results. One can study the tail behavior of the underlying model by truncating the lower, upper or both points. For more information, Abid (2016) double truncated the Fréchet distribution and simulate the model to recognize the performance of the estimates. Abid and Abdulrazak (2017) truncated the Fréchet, Fréchet Uniform and Fréchet Exponential distributions and discussed their strength-stress models as well. Castillo et al. (2018) discussed the half normal distribution by considering zero as a truncation point for $x>0$ and fit it to two-lifetime datasets.

The present distribution is initiated on this motivation that it has not been studied earlier and will present more flexible estimates on the skewed datasets. Furthermore, it is applicable to many diverse problems other than income and wealth studies.

Rest of the article is arranged into several sections as follows: CDF, PDF, graphical representation alongside special cases are developed in Section 2. In Section 3, we illustrate the moments and various reliability measures in Section 4. Quantiles function along with several descriptive statistics, Rényi entropy, The Mellin 
transformation and order statistics is discussed in Section 5. Estimation of the parameters by the maximum likelihood, the study of simulation and application is developed in Section 6 and lastly, the conclusion is reported in Section 7.

\section{Double Truncated Transmuted Fréchet Distribution}

Here we establish a model by applying the technique of double truncation to Transmuted Fréchet distribution, originally developed by Mahmoud and Mandouh (2013). It is referred to a Double Truncated Transmuted Fréchet (DTTF) distribution. The CDF of DTTF distribution is followed by

$$
F(x)=\frac{\left[(1+\lambda) e^{-\alpha x^{-\beta}}-\lambda e^{-2 \alpha x^{-\beta}}\right]-\left[(1+\lambda) e^{-\alpha m^{-\beta}}-\lambda e^{-2 \alpha m^{-\beta}}\right]}{\left[(1+\lambda) A-\lambda A^{2}\right]-\left[(1+\lambda) B-\lambda B^{2}\right]},
$$

and PDF

$$
f(x)=\frac{\left[(1+\lambda) \alpha \beta x^{-\beta-1} e^{-\alpha x^{-\beta}}-2 \alpha \beta \lambda x^{-\beta-1} e^{-2 \alpha x^{-\beta}}\right]}{\left[(1+\lambda) A-\lambda A^{2}\right]-\left[(1+\lambda) B-\lambda B^{2}\right]}
$$

where $x>0, \alpha, \beta>0,|\lambda|<1, A=e^{-\alpha \mathrm{g}^{-\beta}}, B=e^{-\alpha m^{-\beta}}, \boldsymbol{m}$ and $\boldsymbol{g}$ are lower and upper truncation points.

Fig.1 and Fig.2 illustrate the reasonable shapes of CDF and PDF for selected values of the parameters $\alpha, \beta$ and $\lambda$.

CDF Plot

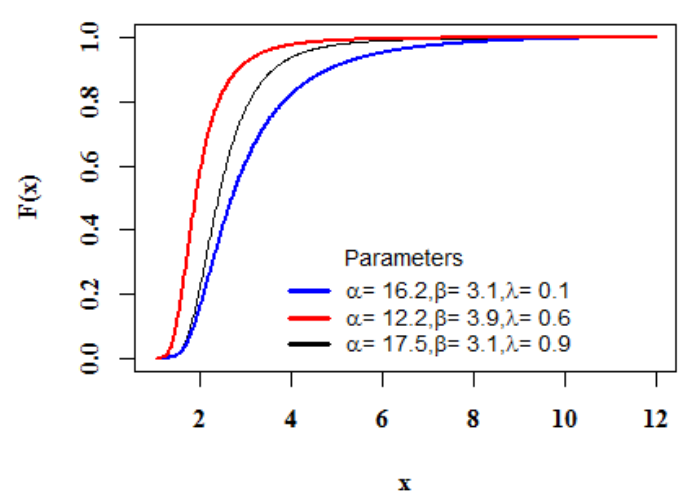

Fig. 1
PDF Plot

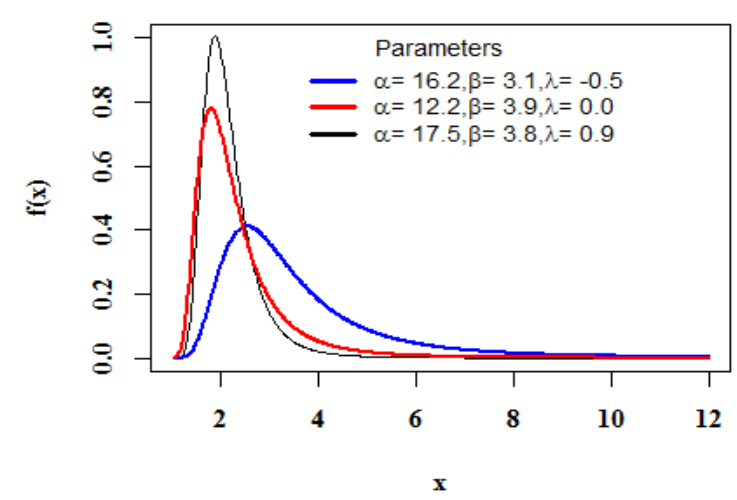

Fig. 2 


\section{Table 1}

Sub-Models of DTTF Distribution

\begin{tabular}{ccccccc}
\hline Models & $\boldsymbol{\alpha}$ & $\boldsymbol{\beta}$ & $\boldsymbol{\lambda}$ & $\boldsymbol{m}$ & $\mathbf{g}$ & Author \\
DTrF & $\alpha$ & $\beta$ & 0 & $m$ & $\mathrm{G}$ & Abid (2016) \\
TF & $\alpha$ & $\beta$ & $\lambda$ & 0 & Inf & Mahmoud and Mandouh (2013) \\
F & $\alpha$ & $\beta$ & 0 & 0 & Inf & Fréchet (1924)
\end{tabular}

$\mathrm{D}=$ Double, $\mathrm{Tr}=$ Truncation, $\mathrm{T}=$ Transmutation, $\mathrm{F}=$ Frechet

\section{Properties of DTTF Distribution}

\subsection{Theorem:}

Suppose the r.v. $X \sim \operatorname{DTTF}(x ; \alpha, \beta, m, \mathrm{~g})$, subsequently, $r$-th raw moment of the Double Truncated Transmuted Fréchet distribution is given by

$$
\begin{aligned}
\mu_{r}^{\prime}= & -\frac{\alpha^{\frac{r}{\beta}}(1+\lambda)}{C}\left[\Gamma\left(1-\frac{r}{\beta}, \alpha m^{-\beta}\right)-\Gamma\left(1-\frac{r}{\beta}, \alpha \mathrm{g}^{-\beta}\right)\right] \\
& +\frac{2 \lambda \alpha^{\frac{r}{\beta}}}{C}\left[\Gamma\left(1-\frac{r}{\beta}, 2 \alpha m^{-\beta}\right)-\Gamma\left(1-\frac{r}{\beta}, 2 \alpha \mathrm{g}^{-\beta}\right)\right],
\end{aligned}
$$

where $x>0, \alpha, \beta>0,|\lambda|<1, C=\left[(1+\lambda) A-\lambda A^{2}\right]-\left[(1+\lambda) B-\lambda B^{2}\right], A=e^{-\alpha \mathrm{g}^{-\beta}}$ and $B=e^{-\alpha m^{-\beta}}, \boldsymbol{m}$ and $\boldsymbol{g}$ are lower and upper truncation points.

By definition

$$
\mu_{r}^{\prime}=\int x^{r} f(x) d x
$$

by equation (2), $r$-th moment of DTTF distribution is written as

$$
\mu_{r}^{\prime}=\int_{m}^{\mathrm{g}} x^{r} \frac{\left[(1+\lambda) \alpha \beta x^{-\beta-1} e^{-\alpha x^{-\beta}}-2 \alpha \beta \lambda x^{-\beta-1} e^{-2 \alpha x^{-\beta}}\right]}{\left[(1+\lambda) A-\lambda A^{2}\right]-\left[(1+\lambda) B-\lambda B^{2}\right]} d x
$$

say $C=\left[(1+\lambda) A-\lambda A^{2}\right]-\left[(1+\lambda) B-\lambda B^{2}\right]$, equation (3) in simplified form

$$
\begin{gathered}
\mu_{r}^{\prime}=\frac{\alpha \beta(1+\lambda)}{C} \int_{m}^{\mathrm{g}} x^{r-\beta-1} e^{-\alpha x^{-\beta}} d x-\frac{2 \alpha \beta \lambda}{C} \int_{m}^{\mathrm{g}} x^{r-\beta-1} e^{-2 \alpha x^{-\beta}} d x, \\
\mu_{r}^{\prime}=\frac{\alpha \beta(1+\lambda)}{C} I_{1}-\frac{2 \alpha \beta \lambda}{C} I_{2},
\end{gathered}
$$


for $I_{1}$ : suppose $\alpha x^{-\beta}=y \Rightarrow x=\left(\frac{y}{\alpha}\right)^{-\frac{1}{\beta}} \Rightarrow d x=-\frac{1}{\alpha \beta}\left(\frac{y}{\alpha}\right)^{-\frac{1}{\beta}-1} d y$,

limits: $x=m \Rightarrow y=\alpha m^{-\beta}, x=\mathrm{g} \Rightarrow y=\alpha \mathrm{g}^{-\beta}$,

$$
\begin{gathered}
I_{1}=-\frac{1}{\beta} \alpha^{\frac{r}{\beta}-1} \int_{\alpha m^{-\beta}}^{\alpha \mathrm{g}^{-\beta}} y^{-\frac{r}{\beta}} e^{-y} d y, \\
I_{1}=-\frac{1}{\beta} \alpha^{\frac{r}{\beta}-1}\left[\Gamma\left(1-\frac{r}{\beta}, \alpha m^{-\beta}\right)-\Gamma\left(1-\frac{r}{\beta}, \alpha \mathrm{g}^{-\beta}\right)\right] .
\end{gathered}
$$

Following the above procedure, we obtain the simplified form of $I_{2}$

$$
I_{2}=-\frac{1}{\beta}(2 \alpha)^{\frac{r}{\beta}-1}\left[\Gamma\left(1-\frac{r}{\beta}, 2 \alpha m^{-\beta}\right)-\Gamma\left(1-\frac{r}{\beta}, 2 \alpha \mathrm{g}^{-\beta}\right)\right]
$$

hence the $r$-th moment of DTTF distribution is obtained by placing equation (5) and equation (6) in equation (4)

$$
\mu_{r}^{\prime}=\left\{\begin{array}{c}
-\frac{\alpha^{\frac{r}{\beta}}(1+\lambda)}{C}\left[\Gamma\left(1-\frac{r}{\beta}, \alpha m^{-\beta}\right)-\Gamma\left(1-\frac{r}{\beta}, \alpha \mathrm{g}^{-\beta}\right)\right] \\
+\frac{2 \lambda \alpha^{\frac{r}{\beta}}}{C}\left[\Gamma\left(1-\frac{r}{\beta}, 2 \alpha m^{-\beta}\right)-\Gamma\left(1-\frac{r}{\beta}, 2 \alpha \mathrm{g}^{-\beta}\right)\right]
\end{array}\right\}, \text { for } r<\beta,
$$

where $x>0, \alpha, \beta>0,|\lambda|<1, \quad C=\left[(1+\lambda) A-\lambda A^{2}\right]-\left[(1+\lambda) B-\lambda B^{2}\right], \quad A=e^{-\alpha \mathrm{g}^{-\beta}}$ and $\quad B=$ $e^{-\alpha m^{-\beta}}, \boldsymbol{m}$ and $\boldsymbol{g}$ are lower and upper truncation points.

3.2. $r$-th negative moments of DTTF distribution are achieved by replacing $r$ by $-r$ in equation (7)

$$
\mu_{-r}^{\prime}=\left\{\begin{array}{c}
-\frac{\alpha^{\frac{-r}{\beta}}(1+\lambda)}{C}\left[\Gamma\left(1+\frac{r}{\beta}, \alpha m^{-\beta}\right)-\Gamma\left(1+\frac{r}{\beta}, \alpha \mathrm{g}^{-\beta}\right)\right] \\
+\frac{2 \lambda \alpha^{\frac{-r}{\beta}}}{C}\left[\Gamma\left(1+\frac{r}{\beta}, 2 \alpha m^{-\beta}\right)-\Gamma\left(1+\frac{r}{\beta}, 2 \alpha \mathrm{g}^{-\beta}\right)\right]
\end{array}\right\},
$$

where $x>0, \alpha, \beta>0,|\lambda|<1, \quad C=\left[(1+\lambda) A-\lambda A^{2}\right]-\left[(1+\lambda) B-\lambda B^{2}\right], \quad A=e^{-\alpha \mathrm{g}^{-\beta}}$ and $\quad B=$ $e^{-\alpha m^{-\beta}}, \boldsymbol{m}$ and $\boldsymbol{g}$ are lower and upper truncation points.

3.3. Fractional positive moments of DTTF distribution is obtained by replacing $r$ with $(\mathrm{m} / \mathrm{n})$ in equation (7)

$$
\mu_{\left(\frac{m}{n}\right)}^{\prime}=\int_{m}^{\mathrm{g}} x^{\left(\frac{m}{n}\right)} f(x) d x
$$

simplification provide 


$$
\mu_{\left(\frac{m}{n}\right)}^{\prime}=\left\{\begin{array}{c}
-\frac{\alpha^{\frac{m}{n \beta}}(1+\lambda)}{C}\left[\Gamma\left(1-\frac{m}{n \beta}, \alpha m^{-\beta}\right)-\Gamma\left(1-\frac{m}{n \beta}, \alpha \mathrm{g}^{-\beta}\right)\right] \\
+\frac{2 \lambda \alpha^{\frac{m}{n \beta}}}{C}\left[\Gamma\left(1-\frac{m}{n \beta}, 2 \alpha m^{-\beta}\right)-\Gamma\left(1-\frac{m}{n \beta}, 2 \alpha \mathrm{g}^{-\beta}\right)\right]
\end{array}\right\}
$$

3.4. Fractional negative moments of DTTF distribution, just replace $(m / n)$ with $(-m / n)$ in equation $(7)$, we get

$$
\mu_{\left(-\frac{m}{n}\right)}^{\prime}=\left\{\begin{array}{c}
-\frac{\alpha^{-\frac{m}{n \beta}}(1+\lambda)}{C}\left[\Gamma\left(1+\frac{m}{n \beta}, \alpha m^{-\beta}\right)-\Gamma\left(1+\frac{m}{n \beta}, \alpha \mathrm{g}^{-\beta}\right)\right] \\
+\frac{2 \lambda \alpha^{\frac{-m}{n \beta}}}{C}\left[\Gamma\left(1+\frac{m}{n \beta}, 2 \alpha m^{-\beta}\right)-\Gamma\left(1+\frac{m}{n \beta}, 2 \alpha \mathrm{g}^{-\beta}\right)\right]
\end{array}\right\} .
$$

3.5. Lower incomplete moments of DTTF distribution, we replace the upper limit $\mathrm{g}$ to $w$ in equation (7)

$$
M_{r}(w)=E_{X \leq w}\left(x^{r}\right)=\int_{m}^{w} x^{r} f(x) d x
$$

hence reduced and simplified form of lower incomplete moments is given by

$$
M_{r}(w)=\left\{\begin{array}{c}
-\frac{\alpha^{\frac{r}{\beta}}(1+\lambda)}{C}\left[\Gamma\left(1-\frac{r}{\beta}, \alpha m^{-\beta}\right)-\Gamma\left(1-\frac{r}{\beta}, \alpha w^{-\beta}\right)\right] \\
+\frac{2 \lambda \alpha^{\frac{r}{\beta}}}{C}\left[\Gamma\left(1-\frac{r}{\beta}, 2 \alpha m^{-\beta}\right)-\Gamma\left(1-\frac{r}{\beta}, 2 \alpha w^{-\beta}\right)\right]
\end{array}\right\} .
$$

3.6. Upper incomplete moments of DTTF distribution are obtained by incorporating equation (7)

$$
M_{S}(w)=E_{X>w}\left(x^{r}\right)=\int_{w}^{\mathrm{g}} x^{r} f(x) d x
$$

hence upper incomplete moments of DTTF distribution is followed by

$$
M_{S}(w)=\left\{\begin{array}{c}
-\frac{\alpha^{\frac{r}{\beta}}(1+\lambda)}{C}\left[\Gamma\left(1-\frac{r}{\beta}, \alpha w^{-\beta}\right)-\Gamma\left(1-\frac{r}{\beta}, \alpha \mathrm{g}^{-\beta}\right)\right] \\
+\frac{2 \lambda \alpha^{\frac{r}{\beta}}}{c}\left[\Gamma\left(1-\frac{r}{\beta}, 2 \alpha w^{-\beta}\right)-\Gamma\left(1-\frac{r}{\beta}, 2 \alpha \mathrm{g}^{-\beta}\right)\right]
\end{array}\right\}
$$

3.7. Factorial moments of DTTF distribution, we achieve by equation (7)

$$
E[X]_{n}=\sum_{r=0}^{n} \varphi_{r} \mu_{r}^{\prime}
$$




$$
E[X]_{n}=\sum_{r=0}^{n} \varphi_{r}\left\{\begin{array}{c}
-\frac{\alpha^{\frac{r}{\beta}}(1+\lambda)}{C}\left[\Gamma\left(1-\frac{r}{\beta}, \alpha m^{-\beta}\right)-\Gamma\left(1-\frac{r}{\beta}, \alpha \mathrm{g}^{-\beta}\right)\right] \\
+\frac{2 \lambda \alpha^{\frac{r}{\beta}}}{C}\left[\Gamma\left(1-\frac{r}{\beta}, 2 \alpha m^{-\beta}\right)-\Gamma\left(1-\frac{r}{\beta}, 2 \alpha \mathrm{g}^{-\beta}\right)\right]
\end{array}\right\},
$$

where

$[X]_{i}=X(X+1)(X+2) \ldots(X+i-1), \varphi_{r}$ is the Stirling number of first kind , $C=[(1+\lambda)(A-B)]-$ $\left[\lambda\left(B-B^{2}\right)\right], A=e^{-\alpha \mathrm{g}^{-\beta}}$ and $B=e^{-\alpha m^{-\beta}}, \boldsymbol{m}$ and $\boldsymbol{g}$ are lower and upper truncation points.

3.8. Moment generating function (m.g.f.) of $r v$. $X$ follow to DTTF distribution is defined as

$$
M_{x}(t)=E\left(e^{t x}\right)=\int_{m}^{\mathrm{g}} e^{t x} f(x) d x
$$

by using series expansion $e^{t x}=\sum_{r=0}^{\infty} \frac{(t x)^{r}}{r !}$, equation (14) can be defined as

$$
\begin{gathered}
M_{x}(t)=\int_{m}^{\mathrm{g}} \sum_{r=0}^{\infty} \frac{(t)^{r}}{r !} x^{r} f(x) d x, \\
M_{x}(t)=\sum_{r=0}^{\infty} \frac{(t)^{r}}{r !}\left\{\begin{array}{c}
-\frac{\alpha^{\frac{r}{\beta}}(1+\lambda)}{C}\left[\Gamma\left(1-\frac{r}{\beta}, \alpha m^{-\beta}\right)-\Gamma\left(1-\frac{r}{\beta}, \alpha \mathrm{g}^{-\beta}\right)\right] \\
+\frac{2 \lambda \alpha^{\frac{r}{\beta}}}{C}\left[\Gamma\left(1-\frac{r}{\beta}, 2 \alpha m^{-\beta}\right)-\Gamma\left(1-\frac{r}{\beta}, 2 \alpha \mathrm{g}^{-\beta}\right)\right]
\end{array}\right\} .
\end{gathered}
$$

3.9. Central moments can be obtained by using a relation between ordinary and central moments. It is defined as

$$
\mu_{r}=E[X-E(X)]^{r}=\sum_{j=0}^{r}\left(\begin{array}{l}
r \\
j
\end{array}\right)(-1)^{j}\left(\dot{\mu}_{1}\right)^{j} \dot{\mu}_{r-j}
$$

based on equation (7), central moments of DTTF distribution

$$
\begin{aligned}
\mu_{r}=\sum_{j=0}^{r}\left(\begin{array}{l}
r \\
j
\end{array}\right)(-1)^{j}\left\{\begin{array}{c}
-\frac{\alpha^{\frac{1}{\beta}}(1+\lambda)}{C}\left[\Gamma\left(1-\frac{1}{\beta}, \alpha m^{-\beta}\right)-\Gamma\left(1-\frac{1}{\beta}, \alpha \mathrm{g}^{-\beta}\right)\right] \\
+\frac{2 \lambda \alpha^{\frac{1}{\beta}}}{C}\left[\Gamma\left(1-\frac{1}{\beta}, 2 \alpha m^{-\beta}\right)-\Gamma\left(1-\frac{1}{\beta}, 2 \alpha \mathrm{g}^{-\beta}\right)\right]
\end{array}\right\} \\
\left\{\begin{array}{c}
-\frac{\alpha^{\frac{r-j}{\beta}}(1+\lambda)}{C}\left[\Gamma\left(1-\frac{r-j}{\beta}, \alpha m^{-\beta}\right)-\Gamma\left(1-\frac{r-j}{\beta}, \alpha \mathrm{g}^{-\beta}\right)\right] \\
+\frac{2 \lambda \alpha^{\frac{r-j}{\beta}}}{C}\left[\Gamma\left(1-\frac{r-j}{\beta}, 2 \alpha m^{-\beta}\right)-\Gamma\left(1-\frac{r-j}{\beta}, 2 \alpha \mathrm{g}^{-\beta}\right)\right]
\end{array}\right\} .
\end{aligned}
$$


3.10. Cumulants generating function based on a relation between ordinary moments and cumulants is defined as

$$
K_{r}=\dot{\mu}_{r}-\sum_{i=1}^{r-1}\left(\begin{array}{l}
r-1 \\
i-1
\end{array}\right) K_{i} \dot{\mu}_{r-i}
$$

cumulants generating function of DTTF distribution can be written as

$$
\begin{gathered}
K_{r}=\left\{\begin{array}{c}
\left.-\frac{\alpha^{\frac{r}{\beta}}(1+\lambda)}{C}\left[\Gamma\left(1-\frac{r}{\beta}, \alpha m^{-\beta}\right)-\Gamma\left(1-\frac{r}{\beta}, \alpha \mathrm{g}^{-\beta}\right)\right]\right\}- \\
+\frac{2 \lambda \alpha^{\frac{r}{\beta}}}{C}\left[\Gamma\left(1-\frac{r}{\beta}, 2 \alpha m^{-\beta}\right)-\Gamma\left(1-\frac{r}{\beta}, 2 \alpha \mathrm{g}^{-\beta}\right)\right]
\end{array}\right\} \\
\sum_{i=0}^{r-1}\left(\begin{array}{l}
r-1 \\
i-1
\end{array}\right) K_{i}\left\{\begin{array}{c}
-\frac{\alpha^{\frac{r-i}{\beta}}(1+\lambda)}{C}\left[\Gamma\left(1-\frac{r-i}{\beta}, \alpha m^{-\beta}\right)-\Gamma\left(1-\frac{r-i}{\beta}, \alpha \mathrm{g}^{-\beta}\right)\right] \\
+\frac{2 \lambda \alpha^{\frac{r-i}{\beta}}}{C}\left[\Gamma\left(1-\frac{r-i}{\beta}, 2 \alpha m^{-\beta}\right)-\Gamma\left(1-\frac{r-i}{\beta}, 2 \alpha \mathrm{g}^{-\beta}\right)\right]
\end{array}\right\} .
\end{gathered}
$$

3.11. Skewness of DTTF distribution is

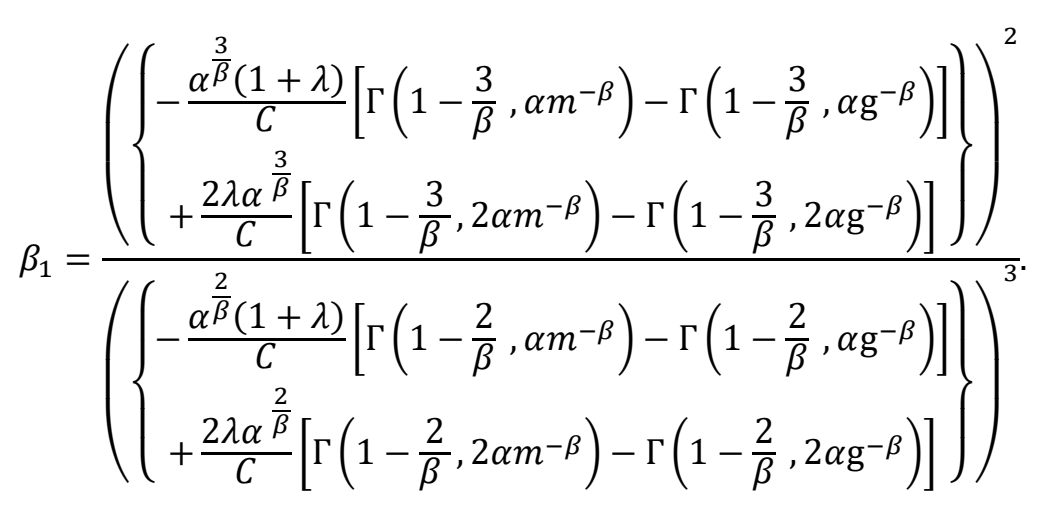

3.12. Kurtosis of DTTF distribution is identified as

$$
\beta_{2}=\frac{\left\{\begin{array}{c}
-\frac{\alpha^{\frac{4}{\beta}}(1+\lambda)}{C}\left[\Gamma\left(1-\frac{4}{\beta}, \alpha m^{-\beta}\right)-\Gamma\left(1-\frac{4}{\beta}, \alpha \mathrm{g}^{-\beta}\right)\right] \\
+\frac{2 \lambda \alpha^{\frac{4}{\beta}}}{C}\left[\Gamma\left(1-\frac{4}{\beta}, 2 \alpha m^{-\beta}\right)-\Gamma\left(1-\frac{4}{\beta}, 2 \alpha \mathrm{g}^{-\beta}\right)\right]
\end{array}\right\}}{\left(\left\{\begin{array}{c}
\left.-\frac{\alpha^{\frac{2}{\beta}}(1+\lambda)}{C}\left[\Gamma\left(1-\frac{2}{\beta}, \alpha m^{-\beta}\right)-\Gamma\left(1-\frac{2}{\beta}, \alpha \mathrm{g}^{-\beta}\right)\right]\right\} \\
+\frac{2 \lambda \alpha^{\frac{2}{\beta}}}{C}\left[\Gamma\left(1-\frac{2}{\beta}, 2 \alpha m^{-\beta}\right)-\Gamma\left(1-\frac{2}{\beta}, 2 \alpha \mathrm{g}^{-\beta}\right)\right]
\end{array}\right\}\right)^{2}}
$$

where $x>0, \alpha, \beta>0,|\lambda|<1, \quad C=\left[(1+\lambda) A-\lambda A^{2}\right]-\left[(1+\lambda) B-\lambda B^{2}\right], \quad A=e^{-\alpha \mathrm{g}^{-\beta}}$ and $B=$ $e^{-\alpha m^{-\beta}}, \boldsymbol{m}$ and $\boldsymbol{g}$ are lower and upper truncation points. 


\section{Reliability Measures of DTTF Distribution}

In reliability engineering, reliability analysis through probability distribution is the most extensively exercised method which pays significant contribution in studying and predicting the survival or hazard life of the component during a particular interval of time.

\subsection{Survival function}

Survival or reliability function is used to measure the risk of occurrence of some event at a specific time. It is denoted by $S(x)$. For DTTF distribution it can written as

$$
S(x)=1-\left(\frac{1}{C}\left\{\left[(1+\lambda) e^{-\alpha x^{-\beta}}-\lambda e^{-2 \alpha x^{-\beta}}\right]-\left[(1+\lambda) e^{-\alpha m^{-\beta}}-\lambda e^{-2 \alpha m^{-\beta}}\right]\right\}\right)
$$

\subsection{Hazard function}

Hazard function $H(x)$ is used to measure the failure rate of some components in a particular period of time $x$. For DTTF distribution it is illustrated by

$$
H(x)=\frac{\left(\frac{1}{C}\left[(1+\lambda) \alpha \beta x^{-\beta-1} e^{-\alpha x^{-\beta}}-2 \alpha \beta \lambda x^{-\beta-1} e^{-2 \alpha x^{-\beta}}\right]\right)}{1-\left(\frac{1}{C}\left\{\left[(1+\lambda) e^{-\alpha x^{-\beta}}-\lambda e^{-2 \alpha x^{-\beta}}\right]-\left[(1+\lambda) e^{-\alpha m^{-\beta}}-\lambda e^{-2 \alpha m^{-\beta}}\right]\right\}\right)}
$$

4.3. Cumulative hazard function $H_{c}(x)$ of DTTF distribution is developed by equation (20)

$$
H_{c}(x)=-\ln \left\{1-\frac{1}{C}\left[(1+\lambda) e^{-\alpha x^{-\beta}}-\lambda e^{-2 \alpha x^{-\beta}}\right]-\left[(1+\lambda) e^{-\alpha m^{-\beta}}-\lambda e^{-2 \alpha m^{-\beta}}\right]\right\}
$$

4.4. Reverse hazard function $H_{r}(x)$ of DTTF distribution is obtained by incorporating equation (1) and equation (20)

$$
H_{r}(x)=\frac{\left(\frac{1}{C}\left[(1+\lambda) \alpha \beta x^{-\beta-1} e^{-\alpha x^{-\beta}}-2 \alpha \beta \lambda x^{-\beta-1} e^{-2 \alpha x^{-\beta}}\right]\right)}{\left(\frac{1}{C}\left\{\left[(1+\lambda) e^{-\alpha x^{-\beta}}-\lambda e^{-2 \alpha x^{-\beta}}\right]-\left[(1+\lambda) e^{-\alpha m^{-\beta}}-\lambda e^{-2 \alpha m^{-\beta}}\right]\right\}\right)}
$$

4.5. Mills ratio $M(x)$ of DTTF distribution is obtained by equation (1) and equation (20)

$$
M(x)=\frac{1-\left(\frac{1}{C}\left\{\left[(1+\lambda) e^{-\alpha x^{-\beta}}-\lambda e^{-2 \alpha x^{-\beta}}\right]-\left[(1+\lambda) e^{-\alpha m^{-\beta}}-\lambda e^{-2 \alpha m^{-\beta}}\right]\right\}\right)}{\left(\frac{1}{C}\left[(1+\lambda) \alpha \beta x^{-\beta-1} e^{-\alpha x^{-\beta}}-2 \alpha \beta \lambda x^{-\beta-1} e^{-2 \alpha x^{-\beta}}\right]\right)}
$$

4.6. Odd function $O(x)$ of DTTF distribution is achieved by equation (2) and equation (20)

$$
O(x)=\frac{\left(\frac{1}{C}\left\{\left[(1+\lambda) e^{-\alpha x^{-\beta}}-\lambda e^{-2 \alpha x^{-\beta}}\right]-\left[(1+\lambda) e^{-\alpha m^{-\beta}}-\lambda e^{-2 \alpha m^{-\beta}}\right]\right\}\right)}{1-\left(\frac{1}{C}\left\{\left[(1+\lambda) e^{-\alpha x^{-\beta}}-\lambda e^{-2 \alpha x^{-\beta}}\right]-\left[(1+\lambda) e^{-\alpha m^{-\beta}}-\lambda e^{-2 \alpha m^{-\beta}}\right]\right\}\right)}
$$


4.7. Elasticity $e(x)$ of DTTF distribution is illustrated by equation (1) and equation (2)

$$
e(x)=\frac{x\left(\frac{1}{C}\left[(1+\lambda) \alpha \beta x^{-\beta-1} e^{-\alpha x^{-\beta}}-2 \alpha \beta \lambda x^{-\beta-1} e^{-2 \alpha x^{-\beta}}\right]\right)}{\left(\frac{1}{C}\left\{\left[(1+\lambda) e^{-\alpha x^{-\beta}}-\lambda e^{-2 \alpha x^{-\beta}}\right]-\left[(1+\lambda) e^{-\alpha m^{-\beta}}-\lambda e^{-2 \alpha m^{-\beta}}\right]\right\}\right)},
$$

where $x>0, \alpha, \beta>0,|\lambda|<1, \quad C=\left[(1+\lambda) A-\lambda A^{2}\right]-\left[(1+\lambda) B-\lambda B^{2}\right], \quad A=e^{-\alpha \mathrm{g}^{-\beta}}$ and $\quad B=$ $e^{-\alpha m^{-\beta}}, \boldsymbol{m}$ and $\boldsymbol{g}$ are lower and upper truncation points.

Possible shapes of survival function, hazard function, cumulative hazard function, reverse hazard function, mills ratio and odd function plots are drafted over various selected combinations of the parameters $\alpha, \beta$ and $\lambda$ display in Fig. 3 to Fig. 8.

\section{Survival Function}

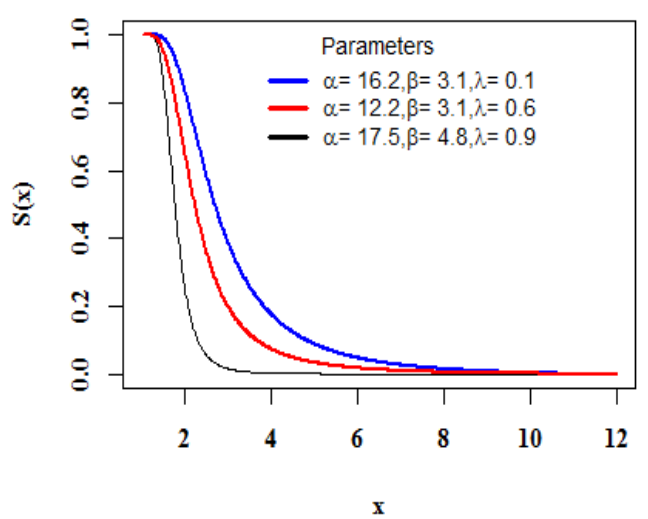

Fig.3 Cumulative Hazard Function

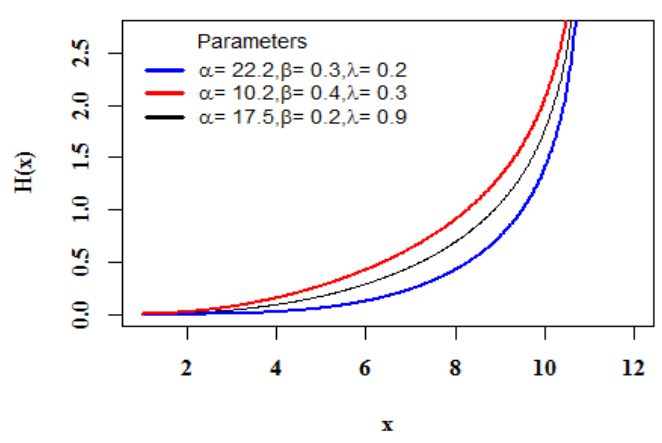

Fig. 5

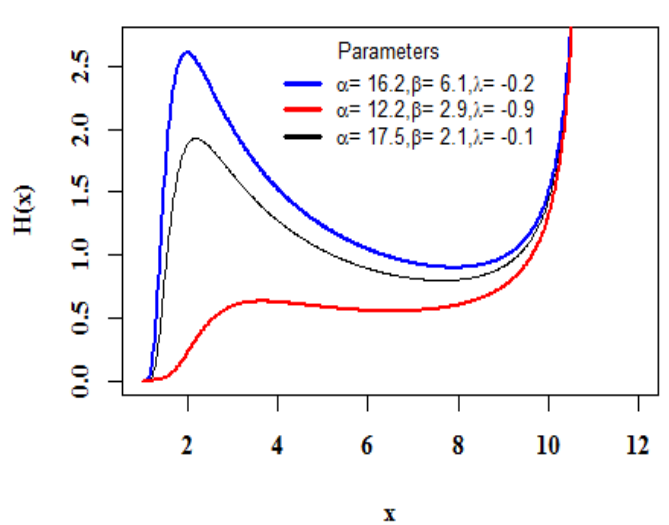

Fig.4

Reverse Hazard Function

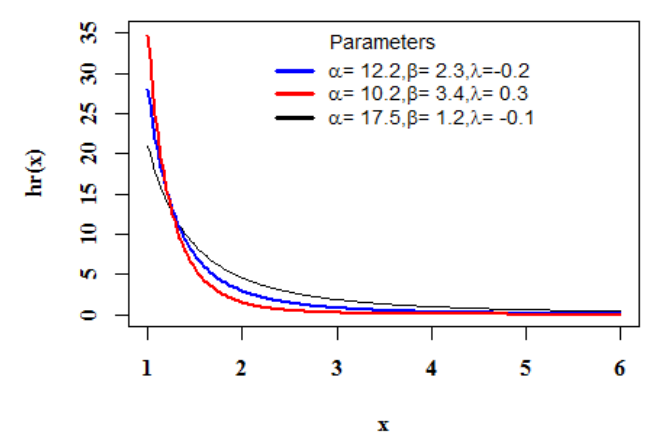

Fig. 6

Mills Ratio

Odd Function 


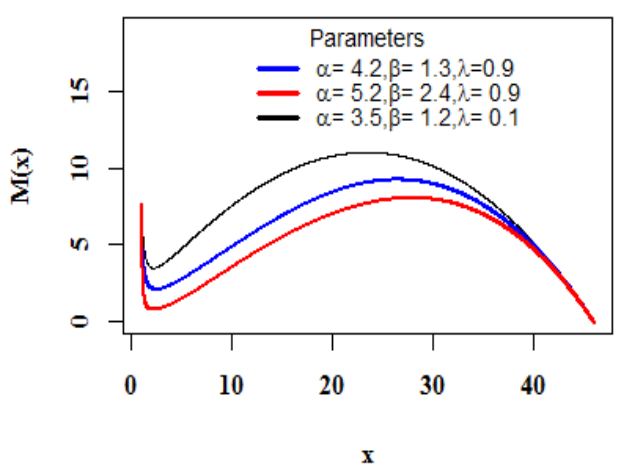

Fig. 7

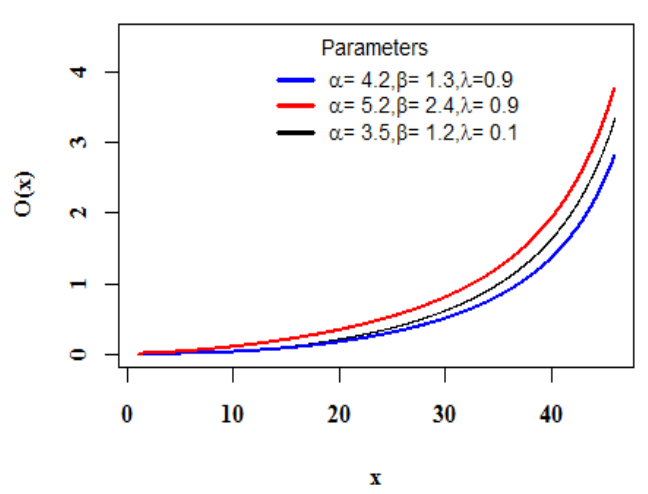

Fig. 8

\section{Quantiles and Descriptive Statisticsof DTTF Distribution}

5.1. Quantile function can be defined as when under investigation CDF is inverted by the method of inversion. It is referred to a quantile function.

$q$-th quantile function of DTTF distribution is given by equation (1)

$$
q=F\left(x_{q}\right)=\frac{\left[(1+\lambda) e^{-\alpha x^{-\beta}}-\lambda e^{-2 \alpha x^{-\beta}}\right]-\left[(1+\lambda) e^{-\alpha m^{-\beta}}-\lambda e^{-2 \alpha m^{-\beta}}\right]}{\left[(1+\lambda) A-\lambda A^{2}\right]-\left[(1+\lambda) B-\lambda B^{2}\right]},
$$

equation (27) can be written as

$$
\begin{gathered}
q=\frac{\left[(1+\lambda) y-\lambda y^{2}\right]-E}{D-E} \text { for } 0 \leq q \leq 1, \\
\lambda y^{2}-(1+\lambda) y+E(1-q)+q D=0,
\end{gathered}
$$

$q^{\text {th }}$ quantile function in reduced form

$$
x_{q}=\left[-\frac{1}{\alpha} \ln \left[\frac{(1+\lambda)-\sqrt{(1+\lambda)^{2}-4 \lambda[q D+E(1-q)]}}{2 \lambda}\right]\right]^{-\frac{1}{\beta}} .
$$

for simplification we suppose $y=e^{-\alpha x^{-\beta}}, E=\left[(1+\lambda) A-\lambda A^{2}\right], \quad D=\left[(1+\lambda) B-\lambda B^{2}\right]$ where $A=$ $e^{-\alpha \mathrm{g}^{-\beta}}, B=e^{-\alpha m^{-\beta}}, \boldsymbol{m}$ and $\boldsymbol{g}$ are lower and upper truncation points.

5.2. Median can easily achieve by placing $q=0.5$ in equation (28)

$$
x_{0.5}=\left[-\frac{1}{\alpha} \ln \left[\frac{(1+\lambda)-\sqrt{(1+\lambda)^{2}-2 \lambda[D+E]}}{2 \lambda}\right]\right]^{-\frac{1}{\beta}} .
$$


To generate random numbers, we suppose that CDF of DTTF distribution follow to uniform distribution $u=U(0$, $1)$.

5.3. Random numbers of DTTF distribution is calculated by

$$
x_{R d}=\left[-\frac{1}{\alpha} \ln \left[\frac{(1+\lambda)-\sqrt{(1+\lambda)^{2}-4 \lambda[u D+E(1-u)]}}{2 \lambda}\right]\right]^{-\frac{1}{\beta}} .
$$

5.4. Harmonic mean of DTTF distribution is achieved as we replace $r$ by -1 in equation (7)

$$
H M=\left\{\begin{array}{c}
-\frac{\alpha^{-\frac{1}{\beta}}(1+\lambda)}{C}\left[\Gamma\left(1+\frac{1}{\beta}, \alpha m^{-\beta}\right)-\Gamma\left(1+\frac{1}{\beta}, \alpha \mathrm{g}^{-\beta}\right)\right] \\
+\frac{2 \lambda \alpha^{-\frac{1}{\beta}}}{C}\left[\Gamma\left(1+\frac{1}{\beta}, 2 \alpha m^{-\beta}\right)-\Gamma\left(1+\frac{1}{\beta}, 2 \alpha \mathrm{g}^{-\beta}\right)\right]
\end{array}\right\} .
$$

5.5. Mean of DTTF distribution is achieved, simply as we replace $r$ by 1 in equation (7)

$$
E(X)=\mu_{1}^{\prime}=\left\{\begin{array}{c}
-\frac{\alpha^{\frac{1}{\beta}}(1+\lambda)}{C}\left[\Gamma\left(1-\frac{1}{\beta}, \alpha m^{-\beta}\right)-\Gamma\left(1-\frac{1}{\beta}, \alpha \mathrm{g}^{-\beta}\right)\right] \\
+\frac{2 \lambda \alpha^{\frac{1}{\beta}}}{C}\left[\Gamma\left(1-\frac{1}{\beta}, 2 \alpha m^{-\beta}\right)-\Gamma\left(1-\frac{1}{\beta}, 2 \alpha \mathrm{g}^{-\beta}\right)\right]
\end{array}\right\} .
$$

5.6. Variance of DTTF distribution may be calculated by incorporating the equation (32) and equation (7)

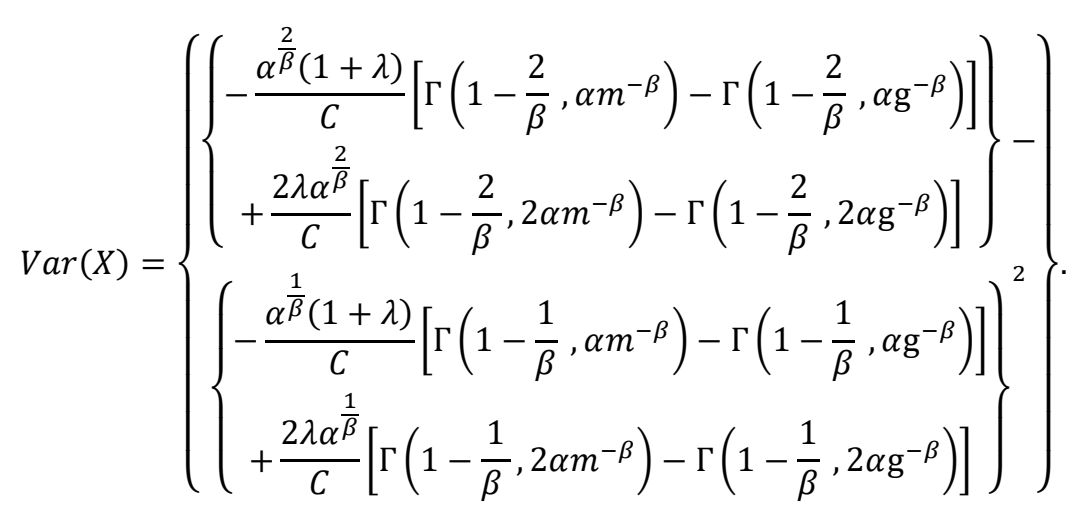

5.7. Mode of DTTF distribution is calculated by taking first derivative of PDF and equate to zero. PDF of DTTF distribution rewrite for the simplification approach

$$
f(x)=\frac{1}{C}\left[(1+\lambda) \alpha \beta x^{-\beta-1} e^{-\alpha x^{-\beta}}-2 \alpha \beta \lambda x^{-\beta-1} e^{-2 \alpha x^{-\beta}}\right],
$$

here we find first derivative and set it to zero 


$$
\frac{1}{C}\left\{\begin{array}{c}
e^{-\alpha x^{-\beta}}(1+\lambda)\left[(\alpha \beta)^{2} x^{(-2 \beta-2)}+\alpha \beta(-\beta-1) x^{(-\beta-2)}\right] \\
-e^{-2 \alpha x^{-\beta}} \lambda\left[(2 \alpha \beta)^{2} x^{(-2 \beta-2)}+2 \alpha \beta x^{(-\beta-2)}(-\beta-1)\right]
\end{array}\right\}=0
$$

since $e^{-\alpha x^{-\beta}}, e^{-2 \alpha x^{-\beta}}$ and $\lambda$ can not be zero for any finite value of $x$

hence

$$
\begin{gathered}
{\left[(\alpha \beta)^{2} x^{(-2 \beta-2)}+\alpha \beta(-\beta-1) x^{(-\beta-2)}\right]=0,} \\
\hat{x}_{1}=\left(\frac{\alpha \beta}{\beta+1}\right)^{\frac{1}{\beta}} .
\end{gathered}
$$

and

$$
\begin{gathered}
-e^{-2 \alpha x^{-\beta}} \lambda\left[(2 \alpha \beta)^{2} x^{(-2 \beta-2)}+2 \alpha \beta x^{(-\beta-2)}(-\beta-1)\right]=0, \\
\hat{x}_{2}=\left(\frac{2 \alpha \beta}{\beta+1}\right)^{\frac{1}{\beta}}
\end{gathered}
$$

thus DTTF distribution is declared as a bimodal distribution

where $x>0, \alpha, \beta>0,|\lambda|<1, \quad C=\left[(1+\lambda) A-\lambda A^{2}\right]-\left[(1+\lambda) B-\lambda B^{2}\right], \quad A=e^{-\alpha \mathrm{g}^{-\beta}}$ and $\quad B=$ $e^{-\alpha m^{-\beta}}, \boldsymbol{m}$ and $\boldsymbol{g}$ are lower and upper truncation points.

\subsection{Entropy of DTTF distribution}

The degree of disorder or unpredictability / randomness in a system is defined as entropy.

By definition, Rényi (1961) entropy is described as

$$
I_{\varphi}(X)=\frac{1}{\varphi-1} \log \int_{0}^{\infty} f^{\varphi}(x) d x \quad \text { for } \varphi>0 \text { and } \varphi \neq 1 .
$$

Rényi entropy of DTTF distribution is obtained by incorporating equation (2)

$$
I_{\varphi}(X)=\frac{1}{\varphi-1} \log \int_{m}^{\mathrm{g}}\left\{\frac{1}{C}\left[\left((1+\lambda) \alpha \beta x^{-\beta-1} e^{-\alpha x^{-\beta}}\right)-\left(2 \alpha \beta \lambda x^{-\beta-1} e^{-2 \alpha x^{-\beta}}\right)\right]\right\}^{\varphi} d x
$$

let's simplify first $f^{\varphi}(x)$

$$
\begin{aligned}
& \text { but } \quad f^{\varphi}(x)=\left\{\frac{1}{c}\left[\left((1+\lambda) \alpha \beta x^{-\beta-1} e^{-\alpha x^{-\beta}}\right)-\left(2 \alpha \beta \lambda x^{-\beta-1} e^{-2 \alpha x^{-\beta}}\right)\right]\right\}^{\varphi} \text {, } \\
& =\left\{\frac{1}{C}(1+\lambda) \alpha \beta x^{-\beta-1} e^{-\alpha x^{-\beta}}\left[1-\frac{2 \lambda e^{-\alpha x^{-\beta}}}{1+\lambda}\right]\right\}^{\varphi},
\end{aligned}
$$




$$
\begin{gathered}
=\frac{1}{C^{\varphi}}\left((1+\lambda) \alpha \beta x^{-\beta-1}\right)^{\varphi} \sum_{j=0}^{\infty}\left\{(-1)^{j}\left(\begin{array}{c}
\varphi \\
j
\end{array}\right)\left(\frac{2 \lambda}{1+\lambda}\right)^{j}\left(e^{-\alpha j x^{-\beta}-\alpha \varphi x^{-\beta}}\right)\right\}, \\
=\left(\frac{(1+\lambda) \alpha \beta}{C}\right)^{\varphi} \sum_{j=0}^{\infty}\left\{(-1)^{j}\left(\begin{array}{c}
\varphi \\
j
\end{array}\right)\left(\frac{2 \lambda}{1+\lambda}\right)^{j} \sum_{k=0}^{\infty} \frac{\left(-\alpha x^{-\beta}(j+\varphi)\right)^{k}}{k !}\right\}, \\
f^{\varphi}(x)=\left(\frac{(1+\lambda) \alpha \beta}{C}\right)^{\varphi} \sum_{j, k=0}^{\infty}\left\{\frac{\alpha^{k}(-1)^{j+k}(j+\varphi)^{k}}{k !}\left(\begin{array}{c}
\varphi \\
j
\end{array}\right)\left(\frac{2 \lambda}{1+\lambda}\right)^{j}\right\} \\
\left.x^{(-\beta(\varphi+k)-\varphi)}\right\}
\end{gathered}
$$

place equation (39) in equation (38), we get

$$
I_{\varphi}(X)=\left(\frac{(1+\lambda) \alpha \beta}{C}\right)^{\varphi}\left(\frac{1}{\varphi-1}\right) \log \int_{m}^{\mathrm{g}} \sum_{j, k=0}^{\infty}\left\{\begin{array}{c}
\frac{(-1)^{j+k}(\alpha(j+\varphi))^{k}}{k !} \\
\left(\begin{array}{c}
\varphi \\
j
\end{array}\right)\left(\frac{2 \lambda}{1+\lambda}\right)^{j} \\
x^{(-\beta(\varphi+k)-\varphi)}
\end{array}\right\} d x
$$

Integrate equation (40) yield the simplified and reduced form of Rényi entropy of DTTF distribution is followed by

$$
I_{\varphi}(X)=\left(\frac{1}{1-\varphi}\right) \log \left[\left(\frac{(1+\lambda) \alpha \beta}{C}\right)^{\varphi} \sum_{j, k=0}^{\infty}\left\{\begin{array}{c}
\frac{(-1)^{j+k}(\alpha(j+\varphi))^{k}}{k !} \\
\left(\begin{array}{c}
\varphi \\
j
\end{array}\right) \\
\left(\frac{2 \lambda}{1+\lambda}\right)^{j} \\
\left(\frac{g^{(1-\beta(\varphi+k)-\varphi)}-m^{(1-\beta(\varphi+k)-\varphi)}}{1-\beta(\varphi+k)-\varphi}\right)
\end{array}\right],\right.
$$

where $x>0, \alpha, \beta>0,|\lambda|<1, \quad C=\left[(1+\lambda) A-\lambda A^{2}\right]-\left[(1+\lambda) B-\lambda B^{2}\right], \quad A=e^{-\alpha \mathrm{g}^{-\beta}}$ and $B=$ $e^{-\alpha m^{-\beta}}, \boldsymbol{m}$ and $\boldsymbol{g}$ are lower and upper truncation points.

\subsection{The Mellin Transformation of DTTF distribution}

In the theory of statistics, the Mellin transformation is well-known since it is a distribution of the product and proportion for independent r.v.'s.

The Mellin transformation is defined as

$$
M_{x}(n)=\mu_{n-1}^{\prime}=E\left(x^{n-1}\right)=\int_{0}^{\infty} x^{n-1} f(x) d x
$$

The Mellin transformation of DTTF distribution, we replace $r$ by $n-1$ in equation (7), we get 


$$
M_{x}(n)=\left\{\begin{array}{c}
-\frac{\alpha^{\frac{n-1}{\beta}}(1+\lambda)}{C}\left[\Gamma\left(1-\frac{n-1}{\beta}, \alpha m^{-\beta}\right)-\Gamma\left(1-\frac{n-1}{\beta}, \alpha \mathrm{g}^{-\beta}\right)\right] \\
+\frac{2 \lambda \alpha^{\frac{n-1}{\beta}}}{C}\left[\Gamma\left(1-\frac{n-1}{\beta}, 2 \alpha m^{-\beta}\right)-\Gamma\left(1-\frac{n-1}{\beta}, 2 \alpha \mathrm{g}^{-\beta}\right)\right]
\end{array}\right\},
$$

where $x>0, \alpha, \beta>0,|\lambda|<1, \quad C=\left[(1+\lambda) A-\lambda A^{2}\right]-\left[(1+\lambda) B-\lambda B^{2}\right], \quad A=e^{-\alpha \mathrm{g}^{-\beta}}$ and $\quad B=$ $e^{-\alpha m^{-\beta}}, \boldsymbol{m}$ and $\boldsymbol{g}$ are lower and upper truncation points.

\subsection{Order Statistics of DTTF distribution}

In reliability analysis and life testing of a component in quality control, order statistics and its moments consider noteworthy measures. Let $X_{1}, X_{2}, X_{3}, \ldots, X_{n}$ be a random sample of size $n$ follows to DTTF distribution and $\left\{X_{(I)}\right.$ $\left.<X_{(2)}<X_{(3)}<\ldots<X_{(n)}\right\}$ be consider as order statistics. The r.v's $X_{(i)}, X_{(1)}, \tilde{X}_{(m)}, X_{(n)}$ be the $i$-th, minimum, median and maximum order statistics of DTTF distribution are followed by

\section{i-th Order Statistic PDF is defined as}

$$
f_{x_{(i)}}(x)=\frac{n !}{(i-1) !(n-i) !}[F(x)]^{i-1}[1-F(x)]^{n-i} f(x)
$$

for $i=1,2,3, \ldots, n$.

by equation (1) and equation (2), $i$-th order statistics PDF of DTTF distribution may obtain by

$$
f_{x_{(i)}}(x)=\frac{n !}{(i-1) !(n-i) !}\left[\begin{array}{c}
\left(\frac{1}{C}\left\{\begin{array}{c}
\left.[1+\lambda) e^{-\alpha x^{-\beta}}-\lambda e^{-2 \alpha x^{-\beta}}\right]- \\
{\left[(1+\lambda) e^{-\alpha m^{-\beta}}-\lambda e^{-2 \alpha m^{-\beta}}\right]}
\end{array}\right\}\right) \\
\times\left(1-\frac{1}{C}\left\{\begin{array}{c}
{\left[(1+\lambda) e^{-\alpha x^{-\beta}}-\lambda e^{-2 \alpha x^{-\beta}}\right]-} \\
{\left[(1+\lambda) e^{-\alpha m^{-\beta}}-\lambda e^{-2 \alpha m^{-\beta}}\right]}
\end{array}\right\}\right)^{n-i} \\
\times \frac{1}{C}\left[(1+\lambda) \alpha \beta x^{-\beta-1} e^{-\alpha x^{-\beta}}-2 \alpha \beta \lambda x^{-\beta-1} e^{-2 \alpha x^{-\beta}}\right]
\end{array}\right] .
$$

\section{Minimum Order Statistic PDF is defined as}

$$
f_{x_{(1)}}(x)=n[1-F(x)]^{n-1} f(x)
$$

minimum order statistic of DTTF distribution is given by

$$
f_{x_{(1)}}(x)=n\left[\begin{array}{c}
\left(1-\frac{1}{C}\left\{\begin{array}{c}
{\left[(1+\lambda) e^{-\alpha x^{-\beta}}-\lambda e^{-2 \alpha x^{-\beta}}\right]-} \\
{\left[(1+\lambda) e^{-\alpha m^{-\beta}}-\lambda e^{-2 \alpha m^{-\beta}}\right]}
\end{array}\right\}\right) \\
\times \frac{1}{C}\left(\left[(1+\lambda) \alpha \beta x^{-\beta-1} e^{-\alpha x^{-\beta}}-2 \alpha \beta \lambda x^{-\beta-1} e^{-2 \alpha x^{-\beta}}\right]\right)
\end{array}\right] .
$$

\section{Maximum Order Statistic PDF is defined as}

$$
f_{x_{(n)}}(x)=n[F(x)]^{n-1} f(x)
$$


maximum order statistic of DTTF distribution is given by

$$
f_{x_{(n)}}(x)=n\left[\begin{array}{c}
\left(\frac{1}{C}\left\{\begin{array}{c}
{\left[(1+\lambda) e^{-\alpha x^{-\beta}}-\lambda e^{-2 \alpha x^{-\beta}}\right]-} \\
{\left[(1+\lambda) e^{-\alpha m^{-\beta}}-\lambda e^{-2 \alpha m^{-\beta}}\right]}
\end{array}\right\}\right)^{n-1} \\
\times \frac{1}{C}\left\{\left[(1+\lambda) \alpha \beta x^{-\beta-1} e^{-\alpha x^{-\beta}}-2 \alpha \beta \lambda x^{-\beta-1} e^{-2 \alpha x^{-\beta}}\right]\right\}
\end{array}\right] .
$$

\section{Median Order Statistic PDF is defined as}

$$
\left.\begin{array}{c}
f_{m}(\tilde{x})=\frac{(2 m+1) !}{m !}[1-F(\tilde{x})]^{m-1}[F(\tilde{x})]^{m} f(\tilde{x}) \\
f_{m}(\tilde{x})=\frac{(2 m+1) !}{m !}\left[\begin{array}{c}
\left(1-\frac{1}{C}\left\{\left[(1+\lambda) e^{-\alpha \tilde{x}-\beta}-\lambda e^{-2 \alpha \tilde{x}-\beta}\right]-\right)\right. \\
\left.\left.\left[(1+\lambda) e^{-\alpha m^{-\beta}}-\lambda e^{-2 \alpha m^{-\beta}}\right]\right\}\right)^{m-1} \\
\times\left(\frac{1}{C}\left\{\left[(1+\lambda) e^{-\alpha \tilde{x}-\beta}-\lambda e^{-2 \alpha \tilde{x}-\beta}\right]-\right)\right. \\
\left.\left[(1+\lambda) e^{-\alpha m^{-\beta}}-\lambda e^{-2 \alpha m^{-\beta}}\right]\right\}
\end{array}\right) \\
\times \frac{1}{C}\left\{\left[(1+\lambda) \alpha \beta \tilde{x}^{-\beta-1} e^{-\alpha \tilde{x}^{-\beta}}-2 \alpha \beta \lambda \tilde{x}^{-\beta-1} e^{-2 \alpha \tilde{x}^{-\beta}}\right]\right\}
\end{array}\right] .
$$

\subsection{Joint Distribution of DTTF distribution}

The joint distribution of $i$-th and $j$-th order statistics of DTTF distribution is

$$
f_{(x, y)_{(i, j)}}(x, y)=E\left[F\left(x_{i}\right)\right]^{i-1}\left[1-F\left(y_{j}\right)\right]^{n-j}\left[F\left(y_{j}\right)-F\left(x_{i}\right)\right]^{j-i-1} f\left(x_{i}\right) f\left(y_{j}\right)
$$

for $i=1,2,3, \ldots, n, j=1,2,3, \ldots, n$ and $E=\frac{n !}{(i-1) !(n-j) !(j-i-1) !}$

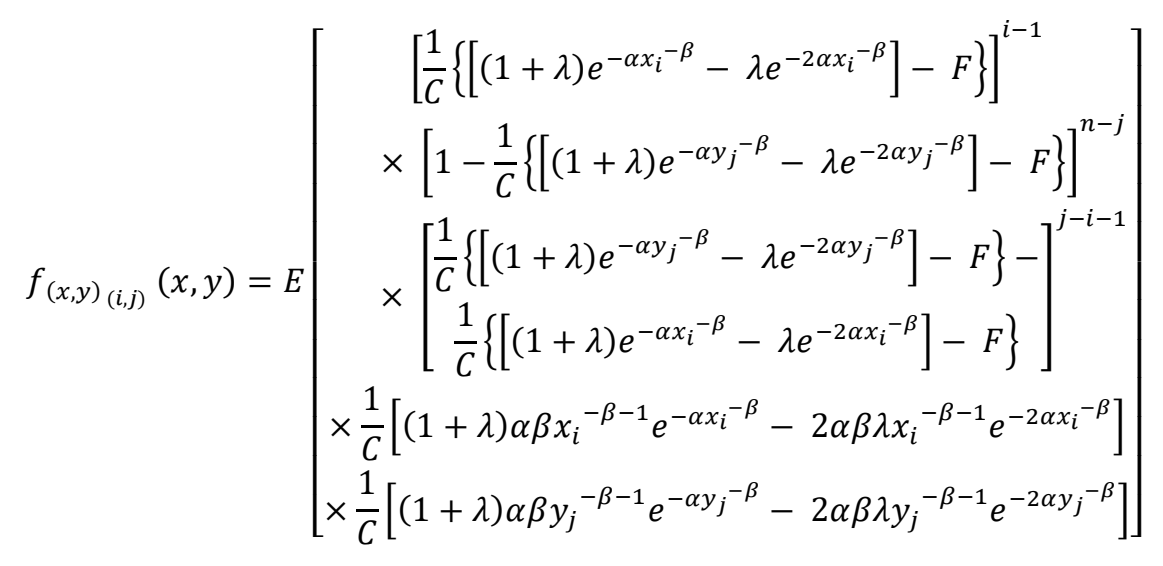

where $x>0, \alpha, \beta>0,|\lambda|<1, C=\left[(1+\lambda) A-\lambda A^{2}\right]-\left[(1+\lambda) B-\lambda B^{2}\right], A=e^{-\alpha \mathrm{g}^{-\beta}}$ and $B=$ $e^{-\alpha m^{-\beta}}, \boldsymbol{m}$ and $\boldsymbol{g}$ are lower and upper truncation points. 


\section{Estimation of Parameters, Simulation Study and Application of DTTF Distribution}

\subsection{Estimation of DTTF distribution}

Parameters of the DTTF distribution are derived by the method of maximum likelihood. Here equation (2) is presented in a simplified way

$$
f(x)=\frac{1}{C} \alpha \beta x^{-\beta-1} e^{-\alpha x^{-\beta}}\left\{(1+\lambda)-2 \lambda e^{-\alpha x^{-\beta}}\right\},
$$

likelihood function of DTTF distribution can be written as

$$
L=\frac{\alpha^{n} \beta^{n}}{C^{n}} \prod_{i=1}^{n}\left(x_{i}\right)^{-\beta-1} \prod_{i=1}^{n}\left(e^{-\alpha x^{-\beta}}\right) \prod_{i=1}^{n}\left\{(1+\lambda)-2 \lambda e^{-\alpha x^{-\beta}}\right\},
$$

$\log$ of equation (48) provides the log-likelihood function of DTTF distribution

$$
\begin{gathered}
l=L L=\mathrm{n}[\ln \alpha+\ln \beta-\ln C]-(\beta+1) \sum \ln x-\alpha \sum x^{-\beta}+ \\
\sum \ln \left[1+\lambda-2 \lambda e^{-\alpha x^{-\beta}}\right]
\end{gathered}
$$

partial derivatives of equation (49) w.r.t $\alpha, \beta, \lambda$ and $\mathrm{C}$ yield

$$
\begin{aligned}
& \frac{\partial l}{\partial \alpha}=\frac{n}{\alpha}-\frac{n}{C} \frac{\partial C}{\partial \alpha}-\sum x^{-\beta}+\sum\left[\frac{2 \alpha \lambda e^{-\alpha x^{-\beta}} x^{-\beta}}{1+\lambda-2 \lambda e^{-\alpha x^{-\beta}}}\right], \\
& \frac{\partial l}{\partial \beta}=\frac{n}{\beta}-\frac{n}{C} \frac{\partial C}{\partial \beta}-\sum \ln x+\alpha \sum x^{-\beta} \ln x+\sum\left[\frac{2 \alpha \lambda e^{-\alpha x^{-\beta}} x^{-\beta} \ln x}{1+\lambda-2 \lambda e^{-\alpha x^{-\beta}}}\right], \\
& \frac{\partial l}{\partial \lambda}=-\frac{n}{C} \frac{\partial C}{\partial \lambda}+\sum\left[\frac{1-2 e^{-\alpha x^{-\beta}}}{1+\lambda-2 \lambda e^{-\alpha x^{-\beta}}}\right],
\end{aligned}
$$

where

$$
\begin{aligned}
& \frac{\partial C}{\partial \alpha}=(1+\lambda)\left[-\mathrm{g}^{-\beta} e^{-\alpha \mathrm{g}^{-\beta}}+m^{-\beta} e^{-\alpha m^{-\beta}}\right]-\lambda\left[-2 \mathrm{~g}^{-\beta} e^{-2 \alpha \mathrm{g}^{-\beta}}+2 m^{-\beta} e^{-2 \alpha m^{-\beta}}\right] \\
& \frac{\partial C}{\partial \beta}=(1+\lambda)\left[\alpha e^{-\alpha \mathrm{g}^{-\beta}} \mathrm{g}^{-\beta} \operatorname{lng}-\alpha e^{-\alpha m^{-\beta}} m^{-\beta} \ln \mathrm{m}\right] \\
& -\lambda\left[2 \alpha e^{-2 \alpha \mathrm{g}^{-\beta}} \mathrm{g}^{-\beta} \operatorname{lng}-2 \alpha e^{-2 \alpha m^{-\beta}} m^{-\beta} \operatorname{lnm}\right], \\
& \frac{\partial C}{\partial \lambda}=\left[e^{-\alpha \mathrm{g}^{-\beta}}-e^{-\alpha m^{-\beta}}\right]-\left[e^{-2 \alpha \mathrm{g}^{-\beta}}-e^{-2 \alpha m^{-\beta}}\right]
\end{aligned}
$$

One can find ML estimates $(\hat{\alpha}, \hat{\beta}, \hat{\lambda})$ by solving these systems of non-linear equations simultaneously present in equation (50), (51), (52). Numerical results are developed by incorporating Statistical software R under the package AdequacyModel. Moreover, the second-order derivatives are required for hypothesis testing and interval estimation. For this, we require $(3 \times 3)$ Fisher information matrix $K(\varphi)$. 


$$
K(\varphi)=\left[\begin{array}{ccc}
\frac{\partial^{2} l}{\partial \alpha^{2}} & \frac{\partial^{2} l}{\partial \alpha \partial \beta} & \frac{\partial^{2} l}{\partial \alpha \partial \lambda} \\
\frac{\partial^{2} l}{\partial \beta \partial \alpha} & \frac{\partial^{2} l}{\partial \beta^{2}} & \frac{\partial^{2} l}{\partial \beta \partial \lambda} \\
\frac{\partial^{2} l}{\partial \lambda \partial \alpha} & \frac{\partial^{2} l}{\partial \lambda \partial \beta} & \frac{\partial^{2} l}{\partial \lambda^{2}}
\end{array}\right] .
$$

Since $\boldsymbol{m}$ and $\boldsymbol{g}$ are the lower and upper truncation points of density function of DTTF distribution, as a result minimum and maximum value of the sample will be considered the estimate of $\boldsymbol{m}$ and $\boldsymbol{g}$.

\subsection{Simulation Study of DTTF distribution}

The study of simulation is conducted to assess the behavior of a finite sample. For DTTF distribution we conduct a small scale experiment and different finite sample of sizes $n=100,200,300,400,500$ and 600 are generated from equation (30). The performance of the estimates are (present in table 1 and table 2) evaluated based on Standard Errors (S.Es). Moreover, numerous statistics are calculated present in table 3 and table 4. Furthermore, 1000 of times simulation is performed to achieve the results and for this entire situation R (Statistical software) is incorporated.

Table 1

MLEs and Standard Errors in parenthesis at various sample sizes for $\alpha=2.5, \beta=2.5, \lambda=0.5$ as left and right truncation points are $m=1.1, g=15.5$

\begin{tabular}{|c|c|c|c|c|c|c|}
\hline \multirow{2}{*}{ Parameters } & \multicolumn{7}{|c|}{ Sample Size } \\
\cline { 2 - 7 } & $\mathbf{1 0 0}$ & $\mathbf{2 0 0}$ & $\mathbf{3 0 0}$ & $\mathbf{4 0 0}$ & $\mathbf{5 0 0}$ & $\mathbf{6 0 0}$ \\
\hline \multirow{2}{*}{$\hat{\alpha}$} & $\begin{array}{c}1.6113 \\
(0.9466)\end{array}$ & $\begin{array}{c}2.6744 \\
(0.6982)\end{array}$ & $\begin{array}{c}2.7767 \\
(0.5433)\end{array}$ & $\begin{array}{c}2.2997 \\
(0.4665)\end{array}$ & $\begin{array}{c}2.3069 \\
(0.3721)\end{array}$ & $\begin{array}{c}2.4979 \\
(0.3378)\end{array}$ \\
\hline \multirow{2}{*}{$\hat{\beta}$} & 2.0335 & 2.6820 & $\begin{array}{c}2.8559 \\
(0.4573)\end{array}$ & $\begin{array}{c}2.5394 \\
(0.3819)\end{array}$ & $\begin{array}{c}2.4008 \\
(0.4685)\end{array}$ & $\begin{array}{c}(0.3789) \\
(0.3599)\end{array}$ \\
\hline \multirow{2}{*}{$\hat{\lambda}$} & $(1.1295)$ & $(0.4793$ \\
& 0.7236 & 0.3078 & 0.3445 & 0.4092 & 0.4772 & 0.4900 \\
$(0.5923)$ & $(0.4533)$ & $(0.3275)$ & $(0.4335)$ & $(0.3147)$ & $(0.2858)$ \\
\hline
\end{tabular}

Table 2

MLEs and Standard Errors in parenthesis at various sample sizes for $\alpha=3.5, \beta=1.5, \lambda=0.5$ as left and right truncation points are $m=1.1, g=75.5$

\begin{tabular}{|c|c|c|c|c|c|c|}
\hline \multirow{2}{*}{ Parameters } & \multicolumn{7}{|c|}{ Sample Size } \\
\cline { 2 - 8 } & $\mathbf{1 0 0}$ & $\mathbf{2 0 0}$ & $\mathbf{3 0 0}$ & $\mathbf{4 0 0}$ & $\mathbf{5 0 0}$ & $\mathbf{6 0 0}$ \\
\hline \multirow{2}{*}{$\hat{\alpha}$} & 2.7837 & 4.1905 & 3.8676 & 3.3731 & 3.6364 & 3.5754 \\
& $(0.7406)$ & $(0.7912)$ & $(0.4956)$ & $(0.4175)$ & $(0.3429)$ & $(0.3191)$ \\
\hline$\hat{\beta}$ & 1.3710 & 1.9228 & 1.7402 & 1.5809 & 1.4952 & 1.5589 \\
& $(0.4501)$ & $(0.2920)$ & $(0.2114)$ & $(0.2371)$ & $(0.1936)$ & $(0.1791)$ \\
\hline$\hat{\lambda}$ & 0.5876 & 0.1601 & 0.3015 & 0.3414 & 0.4198 & 0.4075 \\
& $(0.5634)$ & $(0.5247)$ & $(0.3200)$ & $(0.3873)$ & $(0.2923)$ & $(0.2667)$ \\
\hline
\end{tabular}




\section{Table 3}

Descriptive Statistics at various sample sizes

for $\alpha=2.5, \beta=2.5, \lambda=0.5$ as left and right truncation points are $m=1.1, g=15.5$

\begin{tabular}{|c|c|c|c|c|c|c|}
\hline \multirow{2}{*}{$\begin{array}{c}\text { Descriptive } \\
\text { Statistics }\end{array}$} & $\mathbf{1 0 0}$ & $\mathbf{2 0 0}$ & $\mathbf{3 0 0}$ & $\mathbf{4 0 0}$ & $\mathbf{5 0 0}$ & $\mathbf{6 0 0}$ \\
\cline { 2 - 7 } & 260.90 & 278.80 & 195.20 & 156.40 & 155.30 & 154.30 \\
\hline CV\% & 1.7150 & 1.7320 & 2.7250 & 4.8520 & 4.2340 & 4.7170 \\
\hline Skewness & 5.7280 & 5.8970 & 12.5700 & 39.1800 & 30.5400 & 36.5700 \\
\hline Kurtosis & 162.30 & 361.20 & 478.60 & 678.40 & 886.80 & 1036 \\
\hline AIC & 78.150 & 177.60 & 236.30 & 336.20 & 440.40 & 515.0 \\
\hline- Log-likelihood & & & & & \\
\hline
\end{tabular}

Table 4

Descriptive Statistics at various sample sizes

for $\alpha=3.5, \beta=1.5, \lambda=0.5$ as left and right truncation points are $m=1.1, g=75.5$

\begin{tabular}{|c|c|c|c|c|c|c|}
\hline \multirow{2}{*}{$\begin{array}{c}\text { Descriptive } \\
\text { Statistics }\end{array}$} & \multicolumn{7}{|c|}{ Sample Size } \\
\cline { 2 - 7 } & $\mathbf{1 0 0}$ & $\mathbf{2 0 0}$ & $\mathbf{3 0 0}$ & $\mathbf{4 0 0}$ & $\mathbf{5 0 0}$ & $\mathbf{6 0 0}$ \\
\hline CV\% & 136.70 & 124.30 & 94.80 & 69.92 & 71.55 & 69.50 \\
\hline Skewness & 2.1550 & 2.6620 & 3.8050 & 6.8350 & 5.9070 & 6.6070 \\
\hline Kurtosis & 7.6720 & 12.3500 & 21.8500 & 67.1200 & 52.7800 & 62.5200 \\
\hline AIC & 381.60 & 734.20 & 1134.0 & 1560.0 & 1995.0 & 2360.0 \\
\hline- Log-likelihood & 187.80 & 364.20 & 564.10 & 776.90 & 994.60 & 1177.0 \\
\hline
\end{tabular}

Table 1and tables 2 represent the MLEs along with the standard errors in the parenthesis. We observe the decreasing behavior of standard errors as the size of the sample increases. Furthermore, Table 3, 4 represent various statistics computed on different simulated sample sizes. One can see in table 3, 4 the decreasing trend of coefficient of variation (CV) when the size of sample increases. Moreover, the additional statistics including skewness, kurtosis, Akaike Information Criterion (AIC) and negative Log-likelihood, increase with increase of sample size. All above conditions in the support of proposed model to declare that MLE estimates of DTTF distribution are consistent in their performance and work quit well.

\subsection{Application of DTTF distribution}

In this section, flexibility and potentiality of DTTF distribution is demonstrated by experiencing and integrating two suitable lifetime datasets. The first dataset presented by Ghitany et al. (2008), entitled waiting time (in minutes) before the customer received service in a bank and the second dataset presented by Nadarajah (2007a) entitled, the daily ozone measurements in New York, May-September1973. The results of DTTF distribution and its competing models (Double Truncated Transmuted Fréchet (DTrF), Transmuted Fréchet (TF) and Fréchet (F)) are evaluated based on $-L L$, Akaike Information Criterion (AIC), Bayesian Information Criterion (BIC) and Kolmogorov-Smirnov (K-S). Furthermore, least values of declared statistics lead to consider a model as a reasonable fit for the dataset. 


\section{Competing Models (x > 0):}

Double Truncated Fréchet (DTrF) Distribution: Abid (2016)

$$
G(x)=\frac{e^{-\alpha x^{-\beta}}-e^{-\alpha m^{-\beta}}}{e^{-\alpha \mathrm{g}^{-\beta}}-e^{-\alpha m^{-\beta}}} \quad, \text { for } \alpha, \beta>0, m<x \text { and } \mathrm{g}>x
$$

Transmuted Fréchet (TF) Distribution: Mahmoud and Mandouh (2013)

$$
G(x)=(1+\lambda) e^{-\alpha x^{-\beta}}-\lambda e^{-2 \alpha x^{-\beta}}, \text { for } \alpha, \beta>0 \text { and }|\lambda|<1 \text {, }
$$

Fréchet (F) Distribution: Fréchet (1927)

$$
G(x)=e^{-\alpha x^{-\beta}} \quad \text {, for } \alpha, \beta>0
$$

6.3.1 Data Set-1: Waiting time (in minutes) before the customer received service in a bank discussed by Ghitany et al. (2008).

Table 3

Descriptive Statistics

\begin{tabular}{|c|c|c|c|c|c|}
\hline Mean & Median & S.D & Variance & Skewness & Kurtosis \\
\hline 9.8770 & 8.1 & 7.2370 & 52.3700 & 1.4730 & 5.5400 \\
\hline
\end{tabular}

Table 4

\begin{tabular}{|c|c|c|c|c|c|c|c|}
\hline \multirow{2}{*}{ Models } & \multicolumn{3}{|c|}{$\begin{array}{c}\text { Coefficients } \\
\text { (Standard Error) }\end{array}$} & \multicolumn{4}{|c|}{ Information Criterion } \\
\hline & $\widehat{\alpha}$ & $\widehat{\boldsymbol{\beta}}$ & $\hat{\lambda}$ & $-L L$ & AIC & BIC & K-S \\
\hline DTTF & $\begin{array}{c}3.99 \\
(0.94)\end{array}$ & $\begin{array}{c}1.01 \\
(0.12)\end{array}$ & $\begin{array}{l}-0.77 \\
(0.19)\end{array}$ & 319.49 & 644.99 & 651.24 & 0.07 \\
\hline $\mathrm{DT} r \mathrm{~F}$ & $\begin{array}{c}5.52 \\
(0.79)\end{array}$ & $\begin{array}{c}0.87 \\
(0.11)\end{array}$ & - & 320.92 & 645.84 & 651.50 & 0.08 \\
\hline $\mathrm{TF}$ & $\begin{array}{c}4.93 \\
(0.91)\end{array}$ & $\begin{array}{c}1.28 \\
(0.09)\end{array}$ & $\begin{array}{l}-0.76 \\
(0.15)\end{array}$ & 330.63 & 667.27 & 675.09 & 0.10 \\
\hline $\mathrm{F}$ & $\begin{array}{c}6.53 \\
(0.89)\end{array}$ & $\begin{array}{c}1.16 \\
(0.07)\end{array}$ & - & 334.38 & 672.76 & 677.97 & 0.12 \\
\hline
\end{tabular}

Parameter Estimates and Information Criterion

$m=0.8$ and $g=38.5$ be the lower and upper truncation points

6.3.2. Data Set-2: The daily Ozone measurements in New York, May-September 1973 presented by Nadarajah (2007a). 


\section{Table 5}

Descriptive Statistics

\begin{tabular}{|c|c|c|c|c|c|}
\hline Mean & Median & S.D & Variance & Skewness & Kurtosis \\
\hline 42.13 & 31.50 & 32.99 & 1088 & 1.2260 & 4.1840 \\
\hline
\end{tabular}

Table 6

Parameter Estimates and Information Criterion

$m=1$ and $g=168$ be lower and upper truncation points

\begin{tabular}{|c|c|c|c|c|c|c|c|}
\hline \multirow{2}{*}{ Models } & \multicolumn{3}{|c|}{$\begin{array}{c}\text { Coefficients } \\
\text { (Standard Error) }\end{array}$} & \multicolumn{4}{|c|}{ Information Criterion } \\
\hline & $\widehat{\alpha}$ & $\widehat{\boldsymbol{\beta}}$ & $\hat{\lambda}$ & $-L L$ & AIC & BIC & K-S \\
\hline DTTF & $\begin{array}{c}8.48 \\
(1.98)\end{array}$ & $\begin{array}{c}0.82 \\
(0.09)\end{array}$ & $\begin{array}{l}-0.92 \\
(0.08)\end{array}$ & 542.98 & 1091.96 & 1100.22 & 0.07 \\
\hline $\mathrm{DT} r \mathrm{~F}$ & $\begin{array}{l}11.92 \\
(2.27)\end{array}$ & $\begin{array}{c}0.71 \\
(0.08)\end{array}$ & - & 545.50 & 1094.99 & 1100.51 & 0.08 \\
\hline $\mathrm{TF}$ & $\begin{array}{l}13.17 \\
(2.56)\end{array}$ & $\begin{array}{c}1.07 \\
(0.06)\end{array}$ & $\begin{array}{l}-0.92 \\
(0.08)\end{array}$ & 560.12 & 1126.24 & 1134.51 & 0.12 \\
\hline $\mathrm{F}$ & $\begin{array}{l}17.99 \\
(2.99)\end{array}$ & $\begin{array}{c}0.97 \\
(0.06)\end{array}$ & - & 566.38 & 1136.75 & 1142.26 & 0.14 \\
\hline
\end{tabular}

PDF and CDF plots drafted over empirical histogram for dataset -1

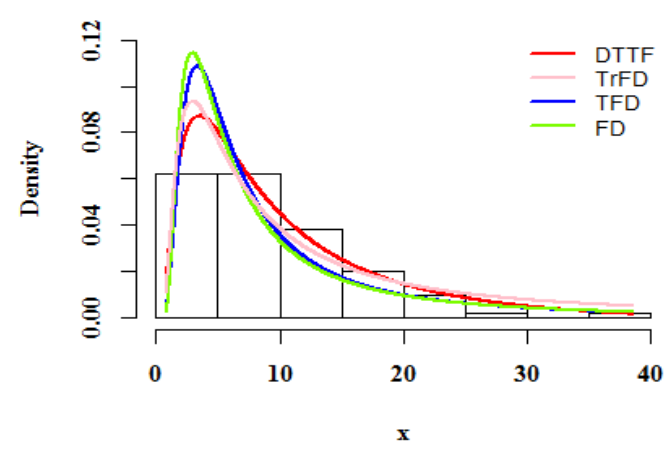

Fig. 9

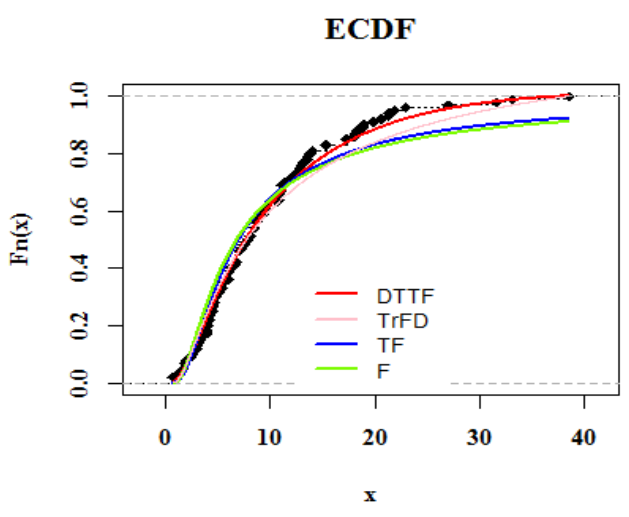

Fig. 10

PDF and CDF plots drafted over empirical histogram for dataset-2 


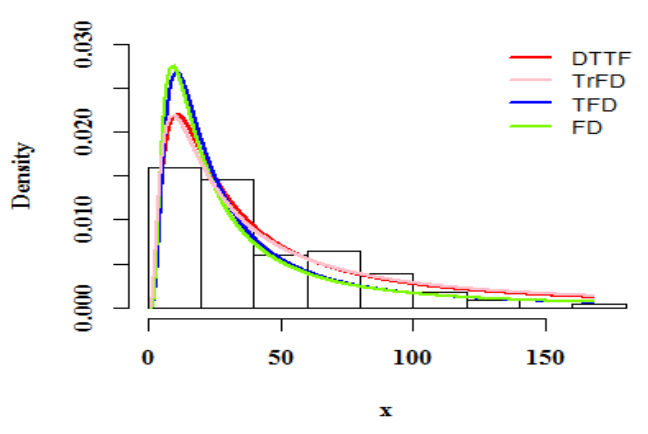

Fig. 11

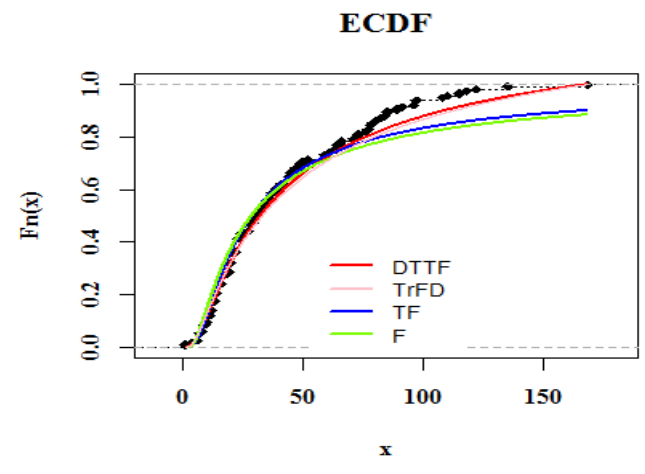

Fig. 12

Total Test Time (TTT) plots for dataset-1 and dataset-2

TTT Plot for dataset- 1

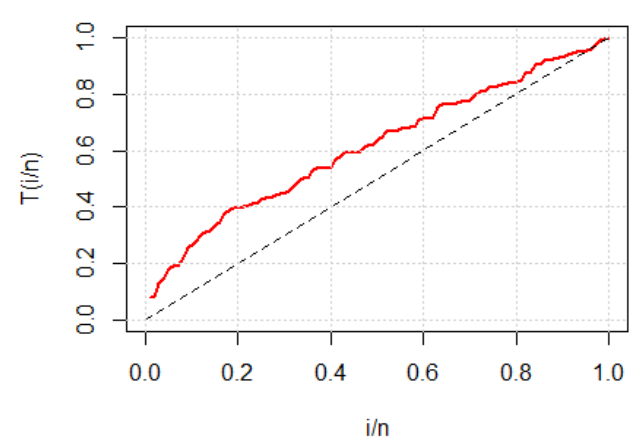

Fig. 13
TTT Plot for dataset- 2

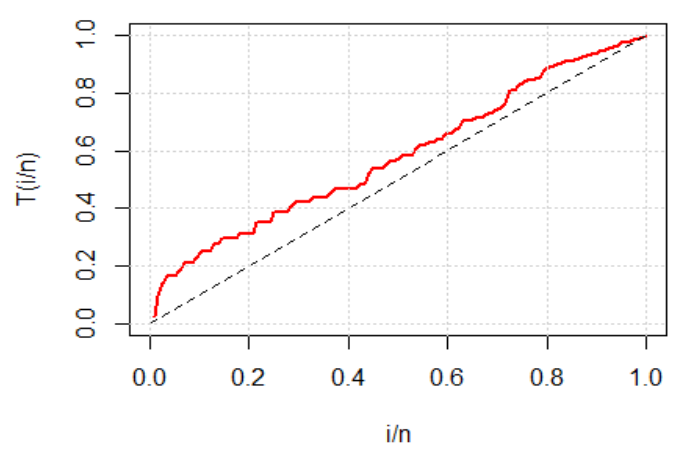

Fig. 14

Various descriptive and empirical results are presented in table 1 to table 6. Table 4, 6 present the empirical results of fitted models comprising Double Truncated Transmuted Fréchet (DTTF), Double Truncated Fréchet $(\mathrm{DT} r \mathrm{~F})$, Transmuted Fréchet (TF) and Fréchet (F) distribution. The Statistical software R is incorporated for the results present in table 1 to 6 . Since the minimum results of $-L L$, AIC, BIC or K-S is the criteria to select the better fit model and results (in table 4,6) are in the support of DTTF distribution. Consequently, we do not hesitate anymore to declare that DTTF distribution is a better fitted model on both the datasets as compared to its competing models. Furthermore, one can see the empirically fitted PDF (Fig. 9 and Fig. 11) and CDF (Fig. 10 and Fig. 12) plots of DTTF distribution display the close fit to the empirical histogram. Fig.13 and Fig. 14 represents the plots of total test time (TTT), proposed by Aarset (1987) may be used as a tool for obtaining empirical behavior of failure rate of the DTTF distribution.

\section{Conclusion}

The present study is conducted to provide supportive and more fixable results than its competing models on skewed datasets. Diverse probabilistic and reliability measures along with Rényi entropy and order statistics are developed and discussed. The method of MLE is suggested to derive the estimates and execution of the estimates is assessed by simulation study. The application of DTTF distribution is illustrated by two real-time datasets. 


\section{References}

[1] Aarset, M. V. (1987). How to identify bathtub hazard rate. IEEE Transactions Reliability, 36, 106108.

[2] Abid, H. S. (2016). Properties of Double-Truncated Fréchet Distribution. American Journal of Applied Mathematics \& Statistics, 4(1), 9-15.

[3] Abayomia, A. and Adeleke, M. (2019). Transmuted Half Normal distribution: Properties and Application. Mathematical Theory \& Modeling, 9(1), 13-26.

[4] Afify, A. Z., Hamedani, G.G., Ghosh, I. and Mead, M.E. (2015). The transmuted Marshall-Olkin Fréchet distribution: properties and applications. International Journal of Statistics \& Probability, 4, 132-184.

[5] Afify, A. Z., Yousof, H.M., Cordeiro, G.M., Nofal, Z.M. and Ahmad, M. (2016a). The Kumaraswamy Marshall-Olkin Fréchet distribution. Journal of ISOSS, 2, 151-168.

[6] Arshad, M. Z., Iqbal, M. Z. and Ahmad, M. (2018). Transmuted Exponentiated Moment Pareto Distribution. Open Journal of Statistics, 08(06), 939-961.

[7] Bhatti, F. A. and Ali, A. (2019). Characterizations of Transmuted Exponentiated Pareto-I Distribution. International Journal of Modern Mathematical Sciences, 17(1), 1-20.

[8] Elrazik Abd, E. M., Altun, E., Afify, A.Z. and Iqbal, M. (2018). A New Three -Parameter Fréchet Distribution: Properties and Applications. Pak. J. Statist., 34(6), 441-458.

[9] Fréchet, M. (1924). Sur la Loi des Erreurs d' Observation. Bulletin de la Soci et e Math ematique de Moscou, 33, 5-8.

[10] Ghitany, M. E., Atieh, B. and Nadarajah, S. (2008). Lindley distribution and its application, Mathematics and Computers in Simulation, 78, 493-506.

[11] Krishna, E., Jose, K.K. and Ristić, M.M. (2013). Applications of Marshall-Olkin Fréchet distribution. Communications in Statistics-Simulation \& Computation, 42, 76-89.

[12] Mansour, M. M., Aryal, G., Afify, A.Z. and Ahmad, M. (2018). The Kumaraswamy Exponentiated Fréchet distribution. Pak. J. Statist., 34(3), 177-193.

[13] Mansour, M.M., Elrazik, E.M.A. and Butt, N. S. (2018). The Exponentiated Marshall-Olkin Fréchet Distribution. Pakistan Journal of Statistics \& Operation Research, 14(1), 57.

[14] Mahmoud, M. R. and Mandouh, R. M. (2013). On the Transmuted Fréchet Distribution. Journal of Applied Sciences Research, 9(10), 5553-5561.

[15] Mead, M. E. and Abd-Eltawab A. R. (2014). A note on Kumaraswamy-Fréchet distribution. Australian Journal of Basic \& Applied Sciences, 8(15), 294-300.

[16] Mead, M. E., Afify, A. Z., Hamedani, G.G. and Ghosh, I. (2017). The Beta Exponentiated Fréchet Distribution. Australian Journal of Statistics, 46, 41-63.

[17] Nadarajah, S. and Kotz, S. (2003). The exponentiated Fréchet distribution. Interstat Electron. J., 17.

[18] Nadarajah, S. (2007a). A truncated inverted beta distribution with application to air pollution data. Stoch. Environ. Res. Risk Assess, doi: 10.1007/s00477-007-0120-7.

[19] Oguntunde, P.E., Khaleel, M.A., Ahmad, M.T. and Okagbue, H. I. (2019). The Gompertz Fréchet distribution: Properties and applications. Cogent Mathematics \& Statistics, 6, 11-12. 
[20] Rényi, A. (1961). Proceeding of the fourth berkely symposium on mathematical statistics and probabilities. First Edition, University of California Press Berkeley.

[21] Shaw, W.T. and Buckley, I. R. (2009). The alchemy of probability distributions: beyond GramCharlier expansions and a skew-kurtotic-normal distribution from a rank transmutation map. arXiv preprint arXiv:0901.0434.

[22] Yousof, H. M., Afify, A. Z., Abd-Ebraheim, El. H. N., Butt, N. S. and Hamedani, G. G. (2016). On Six-Parameter Fréchet Distribution: Properties and Applications, Pakistan Journal of Statistics \& Operation Research, 12(2), 281-299. 